\title{
Spread through air spaces (STAS): a comprehensive update
}

\author{
Arne Warth ${ }^{1,2}$ \\ ${ }^{1}$ Institute of Pathology, Heidelberg University, Heidelberg, Germany; ${ }^{2}$ Translational Lung Research Centre Heidelberg, German Centre for Lung \\ Research, Heidelberg, Germany \\ Correspondence to: Arne Warth. Im Neuenheimer Feld 224, D-69120 Heidelberg, Germany. Email: arne.warth@med.uni-heidelberg.de.
}

\begin{abstract}
The new concept of spread through air spaces (STAS) was introduced for pulmonary adenocarcinomas (ADC) in the 2015 World Health Organization (WHO) classification for lung cancer. Yet available data demonstrate that STAS is of high prognostic impact and associated with specific clinicpathological characteristics. This article provides a comprehensive overview on recent developments in this field.
\end{abstract}

Keywords: Spread through air spaces (STAS); lung cancer; prognosis; metastasis; recurrence

Submitted May 05, 2017. Accepted for publication Jun 01, 2017.

doi: $10.21037 /$ tlcr.2017.06.08

View this article at: http://dx.doi.org/10.21037/tlcr.2017.06.08

\section{Introduction}

Following validation in two large independent cohorts from the US and Germany $(1,2)$, the new concept of spread through air spaces (STAS) was introduced for pulmonary adenocarcinomas (ADC) in the 2015 World Health Organization (WHO) classification for lung cancer and subsequently validated and extended by further studies (3). This article will provide a comprehensive overview on recent developments in this field.

\section{Definition of STAS}

According to the 2015 WHO classification, STAS is defined as "micropapillary clusters, solid nests, or single cells spreading within air spaces beyond the edge of the main tumor". Besides the existing criteria for invasion (a histological subtype other than a lepidic pattern; myofibroblastic stroma associated with invasive tumor cells; vascular or pleural invasion), STAS was thereby established as a fourth category which defines invasion for ADC.

Prior to the definition of STAS, early reports indicated that aerogenous tumor spread and free floating cell clusters from ADC but also metastases from colorectal cancer are unfavorable prognostic features (4-6). Furthermore, Onozato et al. described so-called tumor islands and their adverse prognostic impact, a morphological feature closely related to STAS in 2013 (7). In principal, tumor islands can be separated from STAS by specific criteria (8). Of note, using $3 \mathrm{D}$ reconstruction approaches it was demonstrated that the tumor islands are connected to the main tumor at different levels (9). Subsequent clinicopathological correlations revealed a significant association of tumor islands with smoking, high-grade (solid or micropapillary) predominant pattern, KRAS mutations, and higher nuclear grade. Furthermore, tumor islands were significantly associated with a worse recurrence-free survival (7).

However, due to an overlap of the official definition by the 2015 WHO classification and respective validation studies and owing that the concept of STAS is still evolving, yet available studies applied divergent definitions of STAS and/or related morphological features (Table 1). Interestingly, more or less independent from the different definitions, the clinicopathological associations and the prognostic impact of STAS are highly comparable across all studies (see below).

\section{Invasion of air spaces-a novel diagnostic feature?}

The definition of STAS implies that we accept the invasion of "air" by a tumor as a novel concept, which is clearly unconventional and not compatible with traditional pathological criteria of invasion, where usually the infiltration 
Table 1 Studies applied divergent definitions of STAS and/or related morphological features

\begin{tabular}{|c|c|c|c|c|}
\hline Study & Entity & Number of cases & Stage & Definition of STAS or related features \\
\hline Onozato et al. 2013 (7) & $A D C$ & 261 & $\mid-I I$ & $\begin{array}{l}\text { Isolated, large collection of tumor cells present within alveolar spaces } \\
\text { that lacked well-demarcated micropapillary configuration. The islands } \\
\text { were located at the periphery of the tumor and were separated by at } \\
\text { least a few alveoli }\end{array}$ \\
\hline Kadota et al. 2015 (1) & $A D C$ & 411 & 1 & $\begin{array}{l}\text { Micropapillary clusters, solid nests, or single cells spreading within } \\
\text { air spaces beyond the edge of the main tumor }\end{array}$ \\
\hline Morimoto et al., 2016 (10) & $A D C$ & 67 & I-III & $\begin{array}{l}\text { A group of more than } 3 \text { small clusters containing }<20 \text { nonintegrated } \\
\text { micropapillary tumor cells that were spreading within air spaces, } \\
>3 \mathrm{~mm} \text { apart from the main tumor }\end{array}$ \\
\hline Uruga et al., 2017 (13) & $A D C$ & 208 & 1 & $\begin{array}{l}\text { Micropapillary clusters, solid nests, or single cells spreading within } \\
\text { air spaces beyond the edge of the main tumor. Cases were further } \\
\text { classified as no STAS, low STAS ( } 1-4 \text { clusters of micropapillary or } \\
\text { solid nest-predominant STAS), or high STAS ( } \geq 5 \text { clusters of STAS) }\end{array}$ \\
\hline Dai et al., 2017 (14) & $A D C$ & 554 & 1 & $\begin{array}{l}\text { Tumor cells observed within air spaces in the surrounding lung } \\
\text { parenchyma beyond the edge of the main tumor. It was classified } \\
\text { into three morphologic subtypes: (I) single cells, (II) micropapillary } \\
\text { clusters, and (III) solid nests }\end{array}$ \\
\hline
\end{tabular}

STAS, spread through air spaces; ADC, adenocarcinomas; SCC, squamous cell carcinomas.

of a carcinoma through the basal membrane is considered the border line between an in situ carcinoma and an invasive carcinoma. However, looking on the spectrum of lung carcinomas with its high variability of growth patterns, we, maybe unnoticed, already accepted the invasion of air as a criterion for an aggressive tumor-biological behaviour for years. Papillary or micropapillary patterns of ADC or cell clusters floating within pools of mucin in mucinous or specifically colloid adenocarcinomas are internationally accepted features of an invasive tumor although a stromal invasion or infiltration through the basal membrane is not necessarily present in these cases. Thus, the idea of STAS requires thinking out of the box but in principle the yet established criteria which define invasion in the lungs are not contradictory to this concept. An open question is how long tumor cells can survive following detachment from the main tumor mass and floating free in the air spaces and how they attach again and gain access to energy supply via blood vessels. Looking on intraalveolar macrophages there is a cell population which in principle demonstrates that cell survival within air spaces is possible. The fluid of the alveolar walls or peritumoral edema or hemorrhage might serve as potential sources to bridge the necessary energy supply and oxygen is more or less available ad libitum within the air spaces. From cell culture experiments it is well known that lung cancer cells can survive starvation from cell culture medium for $>12 \mathrm{~h}$, thus providing at least a rough time frame of potential mechanisms involved in STAS in vivo. However, due to the limitations of even modern imaging approaches the precise molecular mechanisms for a detachment of tumor cells, a distribution via air spaces and attachment at distant sites can most likely only be analyzed using animal models, e.g., by transtracheal injection of lung cancer cells in rodents.

\section{Correlation of STAS with clinicopathological parameters}

The concept of STAS was initially validated by two large contemporaneous publications $(1,2)$. In a series of 411 stage I 
ADC it was demonstrated that STAS is a phenomenon usually found in the first alveolar layers close to the tumor but occasionally can be found more than 50 alveolar spaces away from the main tumor. STAS was significantly associated with lymphovascular invasion and high-grade histological pattern (1). The association of STAS to lymphovascular invasion was subsequently validated by others (10). In a series of 569 ADC STAS was significantly associated with male sex, lymph node and distant metastasis, tumor stage, and high-grade histological patterns (2). Further correlations were found with KRAS and $B R A F$ mutations (2). Another recent study concerning STAS in a series of 318 stage I ADC confirmed and extended the yet available data. The authors found significant associations of STAS with the male sex, the median CEA level, smoking status, median maxSUV, solid nodules on computed tomography, p-stage, EGFR wild-type, lymphovascular invasion, and pleural invasion (11). In a series of 209 ADC where the authors aimed to quantify STAS there were statistically significant associations between higher STAS and solid predominant ADC, pleural invasion, lymphatic invasion, vascular invasion, and tumor size $\geq 10 \mathrm{~mm}$ (13).

By analyzing a series of 445 stage I-III lung squamous cell carcinomas (SCC) Lu et al. demonstrate for the first time that STAS is not limited to ADC but is also a significant prognostic parameter for SCC (12). STAS was observed in $30 \%$ of the cases and the frequency increased with stage. Significant associations were identified for several adverse pathologic features including lymphovascular invasion (15).

\section{Prognostic impact of STAS}

Of note, one of the first validation studies on STAS found different survival rates depending on the type of the resection. Whereas STAS was not significantly associated with recurrence in the lobectomy group it was a significant prognosticator for any as well as locoregional recurrence in the limited resection group (1), pointing towards the potential clinicopathological impact of this new feature. In the second validation study, STAS was a significant prognosticator for both overall and disease-free survival even in patients with lobectomy and independent of the extent of STAS. Furthermore, STAS remained a worse prognosticator in multivariate analysis including the predominant pattern but not independent of stage (2). Although the analyzed subgroups were small, this study also demonstrated for the first time that the presence of STAS adds significant prognostic information to the predominant pattern (2), which was further underlined by a subsequent study focusing on the papillary pattern (16). The additive prognostic value of STAS to histological patterns was most recently confirmed by a retrospective study of ADC with a micropapillary component in which the prognostic impact of free tumor cell clusters was analyzed (10). Of note, there was a significant association with recurrencefree survival and mortality (10). More interestingly, the authors demonstrate that the presence of a micropapillary component together with STAS results in a shift of the T stage compared to the same tumors without both features being present. In a further validation study STAS was confirmed as an independent prognostic parameter in multivariate analysis for both overall and recurrence-free survival (11). In a series of 544 ADC with a validation cohort of 541 patients, those with ADCs $3 \mathrm{~cm}$ or smaller and STAS had worse recurrence-free and overall survival than those without STAS. Comparable survival rates were observed in patients with stage IA ADC exhibiting STAS and those with stage IB ADC. Multivariate analysis revealed STAS to be an independent prognostic factor in ADC $3 \mathrm{~cm}$ or smaller. Among patients with ADC larger than 2 to $3 \mathrm{~cm}$, STAS still stratified the prognosis. Moreover, the unfavorable prognosis of patients with ADC larger than 2 to $3 \mathrm{~cm}$ with STAS present was similar to that of patients with stage IB ADC (14).

In an approach to quantify the extent of STAS there was a significant association between increasing amounts of STAS in the tumor periphery and shorter recurrencefree survival in univariate analysis. In multivariate analysis, only STAS remained a significant predictor of survival (13). This points out that, although the distance of STAS from the main tumor does not seem to be of high prognostic relevance in neither for ADC nor SCC $(2,12,14)$, the quantity of STAS might be an important prognostic parameter. Further studies are needed where STAS is analyzed quantitatively to further narrow in on this and to potentially define clinically meaningful cut-offs.

In SCC the cumulative incidence of any, distant, and locoregional recurrence as well as lung cancer-specific death were significantly higher in patients with STAS compared to those without, and these associations remained significant in multivariate analyses. As demonstrated in ADC (2) the extent of STAS was not relevant for prognosis (12). The prognostic impact of STAS in SCC was most recently confirmed in a series of 2016 cases from Japan (17). Thus, beyond budding and cell nest size which has previously 
been identified as prognostically relevant morphological parameters in SCC (18-21), STAS seems to be another morphological feature to allow for a better prognostic stratification of SCC and potentially the establishment of a clinically meaningful grading system in the near future.

An interesting point is that there are first data pointing towards that STAS might be a potential candidate to upgrade the $T$ stage in order to improve the accuracy of the prognosis of early stage ADC. Eguchi et al. demonstrated that the 5-year cumulative incidence of recurrence and cumulative incidence of lung cancer specific death in patients with ADC $2 \mathrm{~cm}$ or smaller was stratified by STAS (22). This is further underlined by a large multinational cohort of >2,000 early stage ADC where STAS together with solid and micropapillary patterns were significant parameters for an optimized patient stratification based on respective nomograms (23). Further analysis revealed that STAS might also be helpful to predict the response to adjuvant treatment (personal experience).

\section{STAS-fact or artifact?}

Up to now all studies on STAS, despite different definitions (Table 1), demonstrate that this novel morphologic feature is of high prognostic value. Thus, STAS should be included into pathological reports. However, it is still debatable whether STAS is an in vivo effect in any instances or potentially an artifact induced by cutting though a tumor with a knife (24). Indeed, data from a recent multicenter study in which a defined protocol for macroscopic tumor dissection was applied demonstrate that the number of free tumor cell clusters within air spaces is increased cut by cut $(25,26)$, pointing towards that at least a certain proportion of STAS might be attributed to the processing of unfixed tissue by the pathologist. On the other side, STAS was reported to be present in tumors which have not been cut in an unfixed state (27). Furthermore, at least a subset of the solid type STAS has been proven to be a direct extension of tumor cells from the main tumor to adjacent air spaces by $3 \mathrm{D}$ reconstruction $(9)$.

Finally, if STAS would only be an artifact induced ex vivo following surgical resection, it is difficult to explain the significant association of STAS with prognosis and recurrence. One may argue that the strong correlation of STAS with lymphovascular invasion is causative for the locoregional and distant recurrence. However, yet available studies demonstrated that STAS was a significant prognosticator independent of lymphovascular invasion
$(1,11)$. So if the prognostic impact and the high association with locoregional recurrence cannot be fully explained by lymphovascular invasion, STAS occurring in situ seems to be the only logic alternative.

With respect to a better separation of STAS from artificial floaters it was suggested to consider only tumor cells as STAS if they appear as detached small clusters within air spaces in a continuous manner from the edge of the tumor, and the distribution is consistent with the overall configuration of the circumferential tumor edge. Haphazardly distributed fragments of tumor with sharp jagged edges might be regarded as artifacts (12). Within this context further studies are clearly warranted to better separate floaters from STAS and evaluate the potential prognostic impact of both.

\section{STAS: spread through a surgeon?}

Given that STAS occurs in situ and is thus causative for tumor recurrence, we must consider all scenarios prior to the material reaching the pathologist. One step back there is a manual surgical procedure where tumors are subjected to mechanical alterations by various surgical tools. Looking specifically on procedures like VATS lobectomy, whole lobes including tumors of different sizes are squeezed through tiny holes in the rigid thoracic wall, which might result in a detachment of tumor cells at the tumor periphery. Furthermore, to localize small tumors within lobes, surgeons use their hands and apply pressure to the lung tissue to feel for resistances. All this undoubtedly results in mechanical stress to a tumor, which might have somewhat discohesive properties and is thus prone to the dissemination of cell clusters from the periphery of the tumor into the adjacent air spaces. Thus, both surgical and pathological handling of resection specimens might be causative for an artificial spread of tumor cells within air spaces beyond the main tumor mass. Even so, the high correlation of STAS to prognosis and recurrence still remains an enigma. One may argue that local recurrence only occurs following wedge resections were STAS is present and detached tumor cells survive within air spaces beyond the stapler margins. However, cases with STAS being present also had a worse prognosis following lobectomy in several studies. Another yet open question is if STAS can be reliably detected in frozen sections, the only option to directly impact the surgical procedure since STAS is not evident in preoperative imaging procedures. Up to now only sparse data are available in this context. Kameda 
et al. reported that STAS could be identified in frozen sections with a sensitivity, specificity, and accuracy of $71 \%$, $92.4 \%$, and $80 \%$, respectively. Clearly these results have to be validated in subsequent studies (28).

Another potential cause for an artificial distribution of tumor cells within air spaces are bronchoscopic or transthoracic tumor biopsies, where also single tumor cells or cell clusters might be detached and then result in a more rapid tumor dissemination through air spaces. Up to now only very limited data are available in this regard. In a series of 146 histologically confirmed cases of lung cancer diagnosed by computed tomography guided transthoracic needle biopsies, there was no increased risk for pleuritis carcinomatosa compared to 112 controls, indicating that at least pleural dissemination seems not to be a problem in this setting. Intrapulmonary tumor spread was not specifically assessed in this study (29).

\section{Conclusions and perspectives}

In summary, STAS is associated with multiple pathologic and clinical features of aggressiveness in both SCC and $\mathrm{ADC}$ and is an important prognostic feature independent of tumor stage and ADC growth patterns. Therefore, STAS might have significant impact for treatment decisions and should be included in pathological reports.

One important next step will be the development of a more precise definition of STAS. For the moment it seems that single tumor cells within air spaces beyond the margins of the main tumor are sufficient to classify a tumor as STAS positive, however, more data concerning the quantitative effect and the distance of STAS from the main tumor and the role of the surgical procedure are warranted. The separation of tumor islands from STAS seems not to be relevant with respect to prognosis. However, based on the current variety of definitions and concepts in the literature, further clarification is needed to determine if "micropapillary STAS" and "isolated tumors cells" as described in the WHO classification differ from the concepts of micropapillary carcinoma occurring at the edge of a tumor and "free tumor clusters" or whether these all represent points in a spectrum of a growth pattern with common behavior. In addition to the need for more clinicopathological and prognostic studies on STAS, more precise criteria to separate ex vivo artifacts from potential in vivo effects are also needed, especially if cases categorized as STAS positive and cases with artificial floaters differ with respect to prognosis. Specifically the identification of molecular mechanisms resulting in STAS and/or in discohesive tumor properties leading to artificial floaters during surgical or pathological tumor manipulation, e.g., alterations of junction proteins (30), will provide in-depth insights if this all represents a continuous phenomenon starting in vivo prior or during surgical procedures and pathological processing or if we can finally clearly separate in vivo effects from artifacts.

A yet still open but important question is also if STAS can reliably be detected in frozen sections, as some studies have demonstrated that the identification of a micropapillary growth pattern at the time of frozen showed high specificity but very low sensitivity $(31,32)$. In conclusion, while the concept of STAS appears to have critical prognostic implications, its refinement and further studies are needed in several areas.

\section{Acknowledgements}

None.

\section{Footnote}

Conflicts of Interest: The author has no conflicts of interest to declare.

\section{References}

1. Kadota K, Nitadori J, Sima CS, et al. Tumor Spread through Air Spaces is an Important Pattern of Invasion and Impacts the Frequency and Location of Recurrences after Limited Resection for Small Stage I Lung Adenocarcinomas. J Thorac Oncol 2015;10:806-14.

2. Warth A, Muley T, Kossakowski CA, et al. Prognostic Impact of Intra-alveolar Tumor Spread in Pulmonary Adenocarcinoma. Am J Surg Pathol 2015;39:793-801.

3. Warth A, Beasley MB, Mino-Kenudson M. Breaking New Ground: The Evolving Concept of Spread through Air Spaces (STAS). J Thorac Oncol 2017;12:176-8.

4. Clayton F. Bronchioloalveolar carcinomas. Cell types, patterns of growth, and prognostic correlates. Cancer 1986;57:1555-64.

5. Shiono S, Ishii G, Nagai K, et al. Predictive factors for local recurrence of resected colorectal lung metastases. Ann Thorac Surg 2005;80:1040-5.

6. Shiono S, Ishii G, Nagai K, et al. Histopathologic prognostic factors in resected colorectal lung metastases. Ann Thorac Surg 2005;79:278-82; discussion 283. 
7. Onozato ML, Kovach AE, Yeap BY, et al. Tumor islands in resected early-stage lung adenocarcinomas are associated with unique clinicopathologic and molecular characteristics and worse prognosis. Am J Surg Pathol 2013;37:287-94.

8. Morales-Oyarvide V, Mino-Kenudson M. Tumor islands and spread through air spaces: Distinct patterns of invasion in lung adenocarcinoma. Pathol Int 2016;66:1-7.

9. Onozato ML, Klepeis VE, Yagi Y, et al. A role of threedimensional (3D)-reconstruction in the classification of lung adenocarcinoma. Anal Cell Pathol (Amst) 2012;35:79-84.

10. Morimoto J, Nakajima T, Suzuki H, et al. Impact of free tumor clusters on prognosis after resection of pulmonary adenocarcinoma. J Thorac Cardiovasc Surg 2016;152:6472.e1.

11. Shiono S, Yanagawa N. Spread through air spaces is a predictive factor of recurrence and a prognostic factor in stage I lung adenocarcinoma. Interact Cardiovasc Thorac Surg 2016;23:567-72.

12. Lu S, Tan KS, Kadota K, et al. Spread Through Air Spaces (STAS) is an Independent Predictor of Recurrence and Lung Cancer Specific Death in Squamous Cell Carcinoma. J Thorac Oncol 2017;12:223-34.

13. Uruga H, Fujii T, Fujimori $S$, et al. Semiquantitative Assessment of Tumor Spread through Air Spaces (STAS) in Early-Stage Lung Adenocarcinomas. J Thorac Oncol 2017;12:1046-51.

14. Dai C, Xie H, Su H, et al. Tumor Spread through Air Spaces Affects the Recurrence and Overall Survival in Patients with Lung Adenocarcinoma $>2$ to $3 \mathrm{~cm}$. J Thorac Oncol 2017;12:1052-60.

15. Yanagawa N, Shiono S, Abiko M, et al. P3.01-002 The clinical impact of spread through air spaces (STAS) in surgically resected pStage I lung squamous cell carcinoma. J Thoracic Oncol 2017;12:S1119-20.

16. Warth A, Muley T, Harms A, et al. Clinical Relevance of Different Papillary Growth Patterns of Pulmonary Adenocarcinoma. Am J Surg Pathol 2016;40:818-26.

17. Kadota K, Kushida Y, Katsuki N, et al. Tumor Spread Through Air Spaces Is an Independent Predictor of Recurrence-free Survival in Patients With Resected Lung Squamous Cell Carcinoma. Am J Surg Pathol 2017;41:1077-86.

18. Weichert W, Kossakowski C, Harms A, et al. Proposal of a prognostically relevant grading scheme for pulmonary squamous cell carcinoma. Eur Respir J 2016;47:938-46.
19. Zhao Y, Shen H, Qiu C, et al. Invasion Types Are Associated With Poor Prognosis in Lung Squamous Carcinoma Patients. Medicine (Baltimore) 2015;94:e1634.

20. Kadota K, Nitadori J, Woo KM, et al. Comprehensive pathological analyses in lung squamous cell carcinoma: single cell invasion, nuclear diameter, and tumor budding are independent prognostic factors for worse outcomes. J Thorac Oncol 2014;9:1126-39.

21. Masuda R, Kijima H, Imamura N, et al. Tumor budding is a significant indicator of a poor prognosis in lung squamous cell carcinoma patients. Mol Med Rep 2012;6:937-43.

22. Eguchi T, Kameda K, Lu S, et al. OA07.06 In Early-Stage Lung Adenocarcinomas, Survival by Tumor Size $(\mathrm{T})$ is Further Stratified by Tumor Spread through Air Spaces. J Thoracic Oncol 2017;12:S270-1.

23. Bains S, Eguchi T, Warth A, et al. PUB016 A multinational cohort validation of procedure-specific nomograms to predict recurrence for small lung adenocarcinomas. J Thoracic Oncol 2017;12:S1455-6.

24. Thunnissen E, Blaauwgeers HJ, de Cuba EM, et al. Ex Vivo Artifacts and Histopathologic Pitfalls in the Lung. Arch Pathol Lab Med 2016;140:212-20.

25. Blaauwgeers HJ, Flieder DB, Warth A, et al. P3.01-009 A Prospective Study of 'Spread through a Knife Surface' (STAKS) in Non-Small Cell Lung Cancer Resection Specimens. J Thorac Oncol 2017;12:S1123-4.

26. Blaauwgeers $\mathrm{H}$, Flieder $\mathrm{D}$, Warth $\mathrm{A}$, et al. A prospective study of loose tissue fragments in non-small cell lung cancer resection specimens: an alternative view to "Spread Through Air Spaces". Am J Surg Pathol 2017;41:1226-30.

27. Lu S, Rekhtman N, Eguchi T, et al. P3.01-029 Cases Demonstrating Spread Through Air Spaces (STAS) Reflects Invasive Growth and Not an Artifact. J Thorac Oncol 2017;12:S1137.

28. Kameda K, Lu S, Eguchi T, et al. MA12.05 Can Tumor Spread through Air Spaces (STAS) in Lung Adenocarcinomas Be Predicted Pre- and Intraoperatively? J Thoracic Oncol 2017;12:S411-2.

29. Flechsig P, Kunz J, Heussel CP, et al. Invasive lung cancer staging: influence of CT-guided core needle biopsy on onset of pleural carcinomatosis. Clin Imaging 2015;39:56-61.

30. Kuner R, Muley T, Meister M, et al. Global gene expression analysis reveals specific patterns of cell junctions in non-small cell lung cancer subtypes. Lung Cancer 2009;63:32-8.

31. Trejo Bittar HE, Incharoen P, Althouse AD, et al. Accuracy 
of the IASLC/ATS/ERS histological subtyping of stage I lung adenocarcinoma on intraoperative frozen sections.

Mod Pathol 2015;28:1058-63.

32. Yeh YC, Nitadori J, Kadota K, et al. Using frozen

Cite this article as: Warth A. Spread through air spaces (STAS): a comprehensive update. Transl Lung Cancer Res 2017;6(5):501-507. doi: 10.21037/tlcr.2017.06.08 section to identify histological patterns in stage I lung adenocarcinoma of $</=3 \mathrm{~cm}$ : accuracy and interobserver agreement. Histopathology 2015;66:922-38. 